\title{
Body mass index is associated with type 2 diabetes mellitus in Chinese elderly
}

This article was published in the following Dove Press journal:

Clinical Interventions in Aging

2 May 2017

Number of times this article has been viewed

\author{
Qianping Zhao' \\ Jari A Laukkanen ${ }^{2,3}$ \\ Qifu $\mathrm{Li}^{4}$ \\ Gang $\mathrm{Li}^{\prime}$
}

'Division of Cardiology, Department of Geriatrics, The First Affiliated Hospital of Chongqing Medical University, Chongqing, People's Republic of China; ${ }^{2}$ Institute of Public Health and Clinical Nutrition, University of Eastern Finland, Kuopio, Finland; ${ }^{3}$ Department of Medicine, Central Finland Central Hospital, Jyväskylä, Finland; ${ }^{4}$ Department of Endocrinology, The First Affiliated Hospital of Chongqing Medical University, Chongqing, People's Republic of China
Correspondence: Gang Li Division of Cardiology, Department of Geriatrics, The First Affiliated Hospital of Chongqing Medical University, No I Youyi Road, Yuzhong, Chongqing 4000I6, People's Republic of China

Tel +86 23 890I 384I

Fax +86 23 688I I487

Email ganglicqmu@126.com
Background: There is limited information on the association between metabolic syndrome components including body mass index (BMI) and type 2 diabetes mellitus in elderly Chinese population. Therefore, we investigated whether components of metabolic syndrome are associated with type 2 diabetes mellitus in elderly.

Methods: A total of 479 hospitalized patients (aged 65-95 years) with recently diagnosed type 2 diabetes mellitus were studied retrospectively in a cross-sectional study and compared with 183 subjects with prediabetes and 62 subjects without glucose metabolism abnormalities.

Results: BMI (24.69 \pm 3.59 versus $23.92 \pm 3.08$ and $\left.23.56 \pm 3.25 \mathrm{~kg} / \mathrm{m}^{2}\right)$, blood pressure, cholesterol, triglyceride, liver enzymes and prevalence of fatty liver were higher in patients with type 2 diabetes mellitus as compared with elderly subjects with prediabetes or normal glucose metabolism separately (all $P<0.05$ ). Multivariable regression analysis showed that BMI was associated positively with insulin resistance and inversely with insulin sensitivity in type 2 diabetes mellitus group (all $P<0.05$ ).

Conclusion: Higher BMI was associated with increased insulin resistance and decreased insulin sensitivity in elderly Asian population with type 2 diabetes mellitus.

Keywords: body mass index, type 2 diabetes, elderly, aging

\section{Introduction}

Morbidity and prevalence of type 2 diabetes mellitus (DM) are increasing in elderly population. The prevalence of type $2 \mathrm{DM}$ in elderly is approximately $22.7 \%$ at present in China. ${ }^{1}$ Global elderly population of type $2 \mathrm{DM}$ has been estimated to increase from 106 million in 2010 to 200 million in 2030. ${ }^{2}$ Type $2 \mathrm{DM}$ is a chronic progressive metabolic disease caused by various pathogenic factors. However, insulin resistance and deficiency of insulin secretion from $\beta$-cells are the main pathogenesis of type $2 \mathrm{DM} .^{3}$ Type 2 DM usually coexists with other components of metabolic syndrome such as high body mass index (BMI), hypertension, hypercholesterolemia, hypertriglyceridemia and fatty liver ${ }^{4-6}$ and increases the risk of all-cause and cardiovascular mortality. ${ }^{7-9}$ High BMI is a predictor of an onset of type 2 DM in older persons aged 65-96 years. ${ }^{10}$ However, a study reported that high BMI was associated with a lower mortality risk in very elderly population aged $\geq 80$ years with type 2 DM. ${ }^{11}$ Some recent studies have demonstrated that a J-shaped association exists between BMI and mortality; high BMI was associated with increased mortality among subjects with type $2 \mathrm{DM}^{8}{ }^{89}$ It is not well known if various metabolic syndrome components such as BMI are related to type $2 \mathrm{DM}$ in Chinese elderly. Therefore, we collected recently diagnosed type 2 DM elderly patients retrospectively in a cross-sectional study in order to investigate the correlation between metabolic syndrome components including BMI and type 2 DM in Chinese elderly. 


\section{Methods}

\section{Patients}

Seven hundred twenty-four elderly patients aged 65-95 years ( 370 males, 354 females) were hospitalized due to comorbidities of diabetes and prediabetes without the use of antidiabetic drugs. The patients' blood glucose concentration was found to be elevated accidentally after hospitalization. Then, they underwent standard $75 \mathrm{~g}$ oral glucose tolerance and insulin release tests from December 2012 to February 2016. Among them, 479 subjects were firstly diagnosed with type $2 \mathrm{DM}$, 183 with prediabetes and 62 with normal glucose metabolism. Data were collected retrospectively in a cross-sectional study from the First Affiliated Hospital of Chongqing Medical University, Chongqing, China.

We excluded patients with type 1 and other types of DM, alcoholic fatty liver, viral hepatitis, hepatic cirrhosis, glomerulonephritis, pyelonephritis, carcinoma, glucocorticoid use, bleeding, acute cardio- or cerebrovascular disease, acute and chronic infection or trauma.

This study was approved by the ethics committee of The First Affiliated Hospital of Chongqing Medical University, Chongqing, China. All participants provided written informed consent.

\section{Diagnosis of type $2 \mathrm{DM}$ and prediabetes}

Diagnosis of type $2 \mathrm{DM}$ and prediabetes was confirmed by oral glucose tolerance and insulin release tests described as follows. After $8 \mathrm{~h}$ fasting period, plasma glucose (fasting plasma glucose [FPG]) and insulin concentrations (fasting plasma insulin [FPI]) were measured. Then, the patients were made to drink $300 \mathrm{~mL}$ syrup containing $75 \mathrm{~g}$ glucose. Postprandial plasma glucose (PPG) and postprandial insulin (PPI) concentrations at 30, 60 and 120 min were determined by glucose oxidase method and radioimmunoassay, respectively. DM, prediabetes and normal glucose metabolism were diagnosed according to the World Health Organization criteria $1998 .{ }^{12} \mathrm{DM}$ is defined as $\mathrm{FPG} \geq 7.0 \mathrm{mmol} / \mathrm{L}$ and/ or $120 \mathrm{~min} P P G \geq 11.1 \mathrm{mmol} / \mathrm{L}$. Prediabetes refers to impaired fasting glucose $(7.0 \mathrm{mmol} / \mathrm{L}>\mathrm{FPG} \geq 6.1 \mathrm{mmol} / \mathrm{L}$ and $120 \mathrm{~min}$ PPG $<7.8 \mathrm{mmol} / \mathrm{L}$ ) and/or impaired glucose tolerance $(\mathrm{FPG}<6.1$ and $11.1 \mathrm{mmol} / \mathrm{L}>120 \mathrm{~min}$ $\mathrm{PPG} \geq 7.8 \mathrm{mmol} / \mathrm{L}$ ). Normal glucose metabolism refers to $6.1 \mathrm{mmol} / \mathrm{L}>\mathrm{FPG} \geq 3.9$ and $7.8 \mathrm{mmol} / \mathrm{L}>120 \mathrm{~min}$ $\mathrm{PPG} \geq 3.9 \mathrm{mmol} / \mathrm{L}^{.2}$

FPI was normal or high and PPI increased to varying degrees compared with baseline, and there was no autoimmune antibody against islet $\beta$-cells and insulin in the recruited elderly with type $2 \mathrm{DM}$ and prediabetes.
Measurement of insulin resistance parameters: homeostasis model assessment (HOMA) and LogHOMA

$\mathrm{HOMA}=\mathrm{FPG} \mathrm{mmol} / \mathrm{L} \times$ FPI $\mu \mathrm{U} / \mathrm{mL} \div 22.5^{13}$

LogHOMA = Logarithm of HOMA ${ }^{14}$

Measurement of insulin sensitivity parameters: $\mathrm{HOMA}^{-1}$, quantitative insulin sensitivity check index (QUICKI) and Gutt's insulin sensitivity index $\left(\mathrm{ISI}_{0,120}\right)$

$\mathrm{HOMA}^{-1}=$ Reciprocal of HOMA ${ }^{14}$

QUICKI $=1 \div(\operatorname{LogFPI} \mu \mathrm{U} / \mathrm{mL}+\log [$ FPG mmol $/ \mathrm{L}$ $\times 18.015])^{15}$

$\mathrm{ISI}_{0,120}=(75,000+[\mathrm{FPG} \mathrm{mmol} / \mathrm{L}-120 \mathrm{~min}$ PPG mmol/L $]$ $\times 0.19 \times$ body weight $\mathrm{kg}) \div(120 \times[\{\mathrm{FPG} \mathrm{mmol} / \mathrm{L}$ $+120 \mathrm{~min}$ PPG mmol/L $\} \div 2] \times \log$ $[\{$ FPI $\mu \mathrm{U} / \mathrm{mL}+120 \mathrm{~min}$ PPI $\mu \mathrm{U} / \mathrm{mL}\} \div 2])^{16}$

Measurement of estimated glomerular filtration rate (eGFR)

For male, serum creatinine (Scr) $>80 \mu \mathrm{mol} / \mathrm{L}$ : eGFR $=141 \times(\mathrm{Scr} \mu \mathrm{mol} / \mathrm{L} \div 88.4 \div 0.9)^{-1.209} \times 0.993^{\text {age, years } 17}$

For male, Scr $\leq 80 \mu \mathrm{mol} / \mathrm{L}$ : eGFR $=141 \times(\mathrm{Scr} \mu \mathrm{mol} / \mathrm{L} \div 88.4 \div 0.9)^{-0.411} \times 0.993^{\text {age, years } 17}$

For female, Scr $>62 \mu \mathrm{mol} / \mathrm{L}$ : eGFR $=144 \times(\mathrm{Scr} \mu \mathrm{mol} / \mathrm{L} \div 88.4 \div 0.7)^{-1.209} \times 0.993^{\text {age, years } 17}$

For female, Scr $\leq 62 \mu \mathrm{mol} / \mathrm{L}$ : eGFR $=144 \times(\mathrm{Scr} \mu \mathrm{mol} / \mathrm{L} \div 88.4 \div 0.7)^{-0.329} \times 0.993^{\text {age, years } 17}$

\section{Measurement of BMI}

BMI $=$ Body weight $\mathrm{kg} \div(\text { Height } \mathrm{m})^{2}$

\section{Measurement of blood lipids}

Total cholesterol (TC), high-density lipoprotein cholesterol (HDL-C) and triglyceride (TG) were determined by cholesterol oxidase, magnesium sulfate precipitation and three color methods, respectively. Low-density lipoprotein cholesterol $($ LDL-C $)=$ TC - HDL-C $-(\mathrm{TG} \div 2.19)$. Apolipoprotein B (apoB) was determined by immune transmission turbidity method.

Measurement of fatty liver and liver enzymes

Fatty liver was diagnosed by color Doppler ultrasound. ${ }^{18}$ Alanyl aminotransferase (ALT), alkaline phosphatase (ALP), 
$\gamma$-glutamyltransferase $(\gamma$-GT) and cholinesterase were determined by rate method.

\section{Statistical analysis}

Continuous parameters were expressed as mean values \pm standard deviation. The statistical differences were evaluated by one-way analysis of variance, followed, in case of significance, by a two-sided Fisher's least significant difference test for multiple comparisons. Categorical data were summarized as percentages and compared using chi-square test. Pearson univariate linear correlation and logistic multivariate regression models were utilized for data analysis. Statistical analysis was performed by SPSS 21.0 software package (IBM Corporation, Armonk, NY, USA). A two-tailed value of $P<0.05$ was considered to be statistically significant.

\section{Results \\ Clinical characteristics}

The age and BMI of the enrolled 724 subjects were $74 \pm 6$ years and $24.39 \pm 3.46 \mathrm{~kg} / \mathrm{m}^{2}$, respectively. Compared with prediabetes and normal groups, the insulin resistance index HOMA and LogHOMA were increased and the insulin sensitivity index $\mathrm{HOMA}^{-1}$, QUICKI and ISI $_{0,120}$ were decreased in the recently diagnosed type $2 \mathrm{DM}$ group (Table 1). There were no differences in age, sex, smoking, drinking, prevalence of coronary heart disease, stroke, hyperthyroidism and gout, and the use of medicines among the three groups (Table 1).

\section{Metabolic syndrome components}

Compared with prediabetes and normal groups, BMI, resting heart rate (HR), prevalence of primary hypertension, systolic and diastolic blood pressure, TC, LDL-C, TG, apoB, prevalence of fatty liver, ALT, ALP, $\gamma$-GT and cholinesterase were higher and HDL-C was lower in patients with recently diagnosed type 2 DM (Table 2).

In univariate correlation analysis, BMI and ALT were correlated positively with insulin resistance index HOMA, and BMI and fatty liver were correlated positively with insulin resistance index LogHOMA among patients with recently diagnosed type $2 \mathrm{DM}$. While BMI and fatty liver were correlated inversely with insulin sensitivity index $\mathrm{HOMA}^{-1}$, BMI was correlated inversely with insulin sensitivity index QUICKI and ISI $_{0,120}$, respectively (Table 3).

Furthermore, in multivariate regression analysis, BMI was only independently directly associated with the insulin resistance index HOMA and LogHOMA (Table 4; Figures 1 and 2) and inversely with sensitivity index $\mathrm{HOMA}^{-1}$, QUICKI and $\mathrm{ISI}_{0,120}$, respectively, among patients with recently diagnosed type 2 DM (Table 4; Figures 3-5).

However, BMI was not associated independently with the insulin resistance and sensitivity index in prediabetes and normal subject groups.

\section{Discussion}

Higher BMI was associated with increased insulin resistance and decreased insulin sensitivity in elderly with recently diagnosed type $2 \mathrm{DM}$. This study shows that higher BMI may be the most important pathogenic factor and associated with type $2 \mathrm{DM}$ in elderly Asian population. These findings were consistent with conclusions from European elderly and Asian population studies. ${ }^{10,19,20}$ They showed that overweight Asian people are still more susceptible to type $2 \mathrm{DM} .{ }^{20}$ Our study confirmed that slightly increased BMI was associated with type $2 \mathrm{DM}$ in elderly Asian population. It could be useful for initiating early interventional measures including balanced diet and regular physical exercise to prevent overweight, obesity and type $2 \mathrm{DM}$ in the elderly population. ${ }^{8}$

Middle-aged adults with elevated resting HR have been reported to have higher risk of incident type $2 \mathrm{DM}$ when they enter old age. ${ }^{21}$ Our present study found that high resting HR was associated with increased insulin resistance and decreased insulin sensitivity in the elderly with recently diagnosed type 2 DM. However, we did not observe an association between resting HR and type $2 \mathrm{DM}$ in this elderly population, suggesting that increased resting HR is not a determinant of type $2 \mathrm{DM}$ among elderly.

A previous meta-analysis concluded that hypertension could predict the risk of incident type $2 \mathrm{DM}$ in adults aged 30-90 years. ${ }^{22}$ This study showed that elevated blood pressure was related to high insulin resistance and low insulin sensitivity in the elderly Chinese population with recently diagnosed type 2 DM. However, the independent association of hypertension with type $2 \mathrm{DM}$ has not been found.

High apoB, LDL-C and TG and low HDL-C have been demonstrated to be associated with the risk of incident type 2 DM. ${ }^{23}$ Previous population studies have shown that hypertriglyceridemia can predict the future risk of incident type 2 DM. ${ }^{24-26}$ We found that TC, LDL-C, TG and apoB were increased among patients with recently diagnosed type 2 DM, while HDL-C was decreased significantly and was related to high insulin resistance and low insulin sensitivity. Nevertheless, this study found that there was no independent association between the abnormal lipid profile and the risk of type 2 DM in elderly, indicating that lipid abnormalities were not risk markers for type $2 \mathrm{DM}$. 
Table I Comparison of clinical characteristics among type 2 diabetes mellitus, prediabetes and normal subject groups

\begin{tabular}{|c|c|c|c|c|}
\hline & Type 2 diabetes mellitus $(n=479)$ & Prediabetes $(n=\mid 83)$ & Normal $(n=62)$ & $P$-value \\
\hline Age, years & $73.83 \pm 6.15$ & $73.35 \pm 5.68$ & $73.08 \pm 5.36$ & 0.484 \\
\hline $\operatorname{Sex}(M / F) n$ & $238 / 241$ & $99 / 84$ & $33 / 29$ & 0.563 \\
\hline $\mathrm{FPG}, \mathrm{mmol} / \mathrm{L}$ & $6.98 \pm 1.68^{\mathrm{a}, \mathrm{b}}$ & $5.67 \pm 0.59^{b}$ & $5.16 \pm 0.42$ & $<0.001$ \\
\hline $30 \mathrm{~min}$ PPG, $\mathrm{mmol} / \mathrm{L}$ & $12.36 \pm 2.94^{\mathrm{a}, \mathrm{b}}$ & $10.06 \pm 1.6 \mathrm{I}^{\mathrm{b}}$ & $8.80 \pm 1.4 \mid$ & $<0.001$ \\
\hline $60 \mathrm{~min}$ PPG, $\mathrm{mmol} / \mathrm{L}$ & $|4.9| \pm 3.19^{\mathrm{a}, \mathrm{b}}$ & $10.80 \pm 2.10^{\mathrm{b}}$ & $8.47 \pm 2.27$ & $<0.001$ \\
\hline $120 \mathrm{~min}$ PPG, $\mathrm{mmol} / \mathrm{L}$ & $|5.8| \pm 3.95^{\mathrm{a}, \mathrm{b}}$ & $9.27 \pm 1.1^{b}$ & $6.50 \pm 0.91$ & $<0.001$ \\
\hline $\mathrm{FPI}, \mu \mathrm{U} / \mathrm{mL}$ & $7.35 \pm 4.96^{\mathrm{b}}$ & $6.46 \pm 4.54$ & $4.95 \pm 2.63$ & 0.083 \\
\hline $30 \mathrm{~min} P P I, \mu U / m L$ & $34.63 \pm 32.64^{\mathrm{a}, \mathrm{b}}$ & $48.78 \pm 32.04$ & $63.1 \pm 47.01$ & $<0.001$ \\
\hline $60 \mathrm{~min} P P I, \mu U / m L$ & $48.03 \pm 45.47^{\mathrm{b}}$ & $59.84 \pm 42.51$ & $70.83 \pm 58.34$ & 0.046 \\
\hline $120 \mathrm{~min} P P I, \mu \mathrm{U} / \mathrm{mL}$ & $69.76 \pm 57.25$ & $72.76 \pm 55.49$ & $44.58 \pm 47.42$ & 0.173 \\
\hline Glycated hemoglobin, \% & $7.05 \pm 1.73^{\mathrm{a}, \mathrm{b}}$ & $6.09 \pm 0.53$ & $6.00 \pm 0.40$ & $<0.001$ \\
\hline HOMA & $2.28 \pm 1.55^{\mathrm{a}, \mathrm{b}}$ & $1.63 \pm 1.26$ & $1.13 \pm 0.62$ & $<0.001$ \\
\hline LogHOMA & $0.27 \pm 0.27^{\mathrm{a}, \mathrm{b}}$ & $0.12 \pm 0.28$ & $-0.0 \mathrm{I} \pm 0.243$ & $<0.001$ \\
\hline $\mathrm{HOMA}^{-1}$ & $0.67 \pm 0.56^{\mathrm{a}, \mathrm{b}}$ & $0.96 \pm 0.89$ & $1.18 \pm 0.75$ & $<0.001$ \\
\hline QUICKI & $0.35 \pm 0.05^{\mathrm{a}, \mathrm{b}}$ & $0.37 \pm 0.04$ & $0.38 \pm 0.03$ & 0.002 \\
\hline$|S|_{0,120}$ & $39.95 \pm 11.32^{\mathrm{a}, \mathrm{b}}$ & $57.8 \mathrm{I} \pm 13.75^{\mathrm{b}}$ & $104.56 \pm 40.35$ & $<0.001$ \\
\hline Smoking, n (\%) & I 44 (30.I) & $53(29.0)$ & $18(29.0)$ & 0.956 \\
\hline Drinking, n (\%) & $90(18.8)$ & $37(20.2)$ & $9(14.5)$ & 0.612 \\
\hline CHD, n (\%) & $79(16.5)$ & $37(20.2)$ & $15(24.2)$ & 0.054 \\
\hline COPD, n (\%) & $57(11.9)$ & $25(13.7)$ & II (I7.7) & 0.404 \\
\hline Stroke, n (\%) & $183(38.2)$ & $81(44.3)$ & $23(37.1)$ & 0.331 \\
\hline Hypothyroidism, n (\%) & $41(18.9)$ & $16(16.0)$ & $9(27.3)$ & 0.359 \\
\hline Hyperthyroidism, n (\%) & $8(3.7)$ & $3(3.0)$ & I (3.0) & 0.945 \\
\hline $\mathrm{TSH}, \mu \mathrm{IU} / \mathrm{mL}$ & $3.57 \pm 9.14$ & $2.38 \pm 2.04$ & $5.60 \pm 17.09$ & 0.189 \\
\hline Gout, n (\%) & $10(2.1)$ & $3(1.6)$ & $\mathrm{I}(\mathrm{I} .6)$ & 0.916 \\
\hline Uric acid, $\mu \mathrm{mol} / \mathrm{L}$ & $341.26 \pm 110.35$ & $335.39 \pm 109.73$ & $322.96 \pm 81.2$ & 0.427 \\
\hline Creatinine, $\mu \mathrm{mol} / \mathrm{L}$ & $80.62 \pm 27.72$ & $81.88 \pm 24.84$ & $79.74 \pm 23.18$ & 0.819 \\
\hline eGFR, mL/min/l.73 m² & $79.36 \pm 19.44$ & $78.60 \pm 17.58$ & $80.10 \pm 16.75$ & 0.838 \\
\hline Hemoglobin, g/L & $130.03 \pm 17.39$ & $|28.62 \pm| 4.82$ & $129.10 \pm 15.22$ & 0.608 \\
\hline Albumin, $g / L$ & $39.24 \pm 4.33$ & $39.6 \pm 4.72$ & $40.46 \pm 4.39$ & 0.115 \\
\hline Fibrinogen, g/L & $3.28 \pm 1.03$ & $3.19 \pm 0.95$ & $3.12 \pm 0.85$ & 0.376 \\
\hline Antiplatelet agents, n (\%) & $273(57.1)$ & 117 (63.9) & $38(61.3)$ & 0.343 \\
\hline Warfarin, n (\%) & $24(5.0)$ & $7(3.8)$ & $5(8.0)$ & 0.863 \\
\hline ACEI, n (\%) & $157(32.8)$ & $47(25.7)$ & $16(25.8)$ & 0.148 \\
\hline ARB, n (\%) & $94(19.6)$ & $38(20.8)$ & $16(25.8)$ & 0.552 \\
\hline$\alpha$ I-blockers, n (\%) & $9(1.9)$ & $7(3.8)$ & $\mathrm{I}(\mathrm{I} .6)$ & 0.310 \\
\hline$\beta$ I-blockers, n (\%) & $130(27.1)$ & $56(30.6)$ & $19(30.6)$ & 0.619 \\
\hline CCB, n (\%) & $187(39.0)$ & $70(38.3)$ & $18(29.0)$ & 0.311 \\
\hline Diuretics, n (\%) & $86(18.0)$ & $29(15.9)$ & $9(14.5)$ & 0.636 \\
\hline Spironolactone, n (\%) & $19(4.0)$ & $8(4.4)$ & $5(8.1)$ & 0.337 \\
\hline Statins, n (\%) & $313(65.3)$ & $122(66.7)$ & $42(67.7)$ & 0.594 \\
\hline Fibrates, n (\%) & $8(1.7)$ & $3(1.6)$ & $0(0)$ & 0.594 \\
\hline Nitrates, n (\%) & $77(16.1)$ & $36(19.7)$ & $12(19.4)$ & 0.496 \\
\hline Trimetazidine, n (\%) & $52(10.9)$ & $21(11.5)$ & $8(12.9)$ & 0.882 \\
\hline Digitalis, n (\%) & $17(3.5)$ & $6(3.3)$ & $2(3.2)$ & $0.98 I$ \\
\hline Finasteride, n (\%) & $14(2.9)$ & $6(3.3)$ & $0(0)$ & 0.390 \\
\hline Allopurine, n (\%) & $2(0.4)$ & $0(0)$ & $0(0)$ & 0.600 \\
\hline Narcaricin, n (\%) & $2(0.4)$ & $2(1.1)$ & $0(0)$ & 0.479 \\
\hline Bicarbonate, n (\%) & $7(1.5)$ & $3(1.6)$ & $2(3.2)$ & 0.614 \\
\hline Thyroxine, n (\%) & $10(2.1)$ & $0(0)$ & $2(3.2)$ & 0.102 \\
\hline
\end{tabular}

Notes: Values are presented as mean \pm SD unless otherwise stated. All the figures are based on nonmissing data. ${ }^{a}<0.05$ versus prediabetes group; ${ }^{b}<0.05$ versus normal group.

Abbreviations: $\mathrm{ACEl}$, angiotensin converting enzyme inhibitor; $\mathrm{ARB}$, angiotensin II receptor inhibitor; $\mathrm{CCB}$, calcium channel blocker; $\mathrm{CHD}$, coronary heart disease; COPD, chronic obstructive pulmonary disease; eGFR, estimated glomerular filtration rate; FPG, fast plasma glucose; FPI, fasting plasma insulin; HOMA, homeostasis model assessment, an insulin resistance index; $\mathrm{HOMA}^{-1}$, reciprocal of HOMA, an insulin sensitivity index; ISI $_{0,120}$, Gutt's insulin sensitivity index; LogHOMA, logarithm of HOMA, an insulin resistance index; PPG, postprandial plasma glucose; PPI, postprandial plasma insulin; QUICKI, quantitative insulin sensitivity check index; SD, standard deviation; $\mathrm{TSH}$, thyroid stimulating hormone; F, female; $\mathrm{M}$, male. 
Table 2 Comparison of metabolic syndrome components among type 2 diabetes mellitus, prediabetes and normal subject groups

\begin{tabular}{|c|c|c|c|c|}
\hline & Type 2 diabetes mellitus $(n=479)$ & Prediabetes $(n=183)$ & Normal $(n=62)$ & $P$-value \\
\hline $\mathrm{BMI}, \mathrm{kg} / \mathrm{m}^{2}$ & $24.69 \pm 3.59^{\mathrm{a}, \mathrm{b}}$ & $23.92 \pm 3.08$ & $23.56 \pm 3.25$ & 0.029 \\
\hline $\mathrm{HR}$, beats/min & $80.28 \pm 14.47^{b}$ & $78.93 \pm 14.5$ & $75.56 \pm 14.84$ & 0.047 \\
\hline Hypertension, n (\%) & $354(73.9)^{\mathrm{a}}$ & $121(66.1)$ & $39(62.9)$ & 0.049 \\
\hline $\mathrm{SBP}, \mathrm{mmHg}$ & $144.67 \pm 23.58^{\mathrm{a}, \mathrm{b}}$ & $137.4 \pm 22.00$ & $132.21 \pm 18.09$ & $<0.001$ \\
\hline $\mathrm{DBP}, \mathrm{mmHg}$ & $79.57 \pm\left. 13.0\right|^{a, b}$ & $76.81 \pm 13.19$ & $75.44 \pm 12.76$ & 0.008 \\
\hline $\mathrm{TC}, \mathrm{mmol} / \mathrm{L}$ & $4.55 \pm 1.07^{a}$ & $4.32 \pm 1.07$ & $4.37 \pm 1.11$ & 0.048 \\
\hline LDL-C, mmol/L & $2.80 \pm 0.90^{\mathrm{a}}$ & $2.6 \pm 0.91$ & $2.65 \pm 0.98$ & 0.056 \\
\hline $\mathrm{HDL}-\mathrm{C}, \mathrm{mmol} / \mathrm{L}$ & $1.18 \pm 0.34^{b}$ & $1.24 \pm 0.30$ & $1.30 \pm 0.05$ & 0.014 \\
\hline $\mathrm{TG}, \mathrm{mmol} / \mathrm{L}$ & $1.8 \mathrm{I} \pm 1.45^{\mathrm{b}}$ & $1.54 \pm 1.38$ & $1.29 \pm 0.91$ & 0.008 \\
\hline ApoB, g/L & $0.91 \pm 0.27^{\mathrm{a}, \mathrm{b}}$ & $0.83 \pm 0.25$ & $0.84 \pm 0.27$ & 0.002 \\
\hline FL, n (\%) & $194(40.6)^{a}$ & $31(17.1)$ & $14(24.0)$ & 0.001 \\
\hline ALT, U/L & $24.06 \pm 17.28^{b}$ & $21.74 \pm 16.11$ & $18.82 \pm 10.47$ & 0.035 \\
\hline ALP, U/L & $81.41 \pm 31.99^{\mathrm{a}, \mathrm{b}}$ & $74.90 \pm 26.98$ & $73.07 \pm 20.08$ & 0.014 \\
\hline$\gamma-\mathrm{GT}, \mathrm{U} / \mathrm{L}$ & $46.38 \pm 69.79^{b}$ & $35.99 \pm 42.49$ & $27.99 \pm 22.7$ & 0.028 \\
\hline CE, U/L & $7,678.98 \pm 1,938.23^{a}$ & $7,307.64 \pm 651.60$ & $7,517.77 \pm 2,289.30$ & 0.047 \\
\hline
\end{tabular}

Notes: Values presented as mean \pm SD unless otherwise stated. a $P<0.05$ versus prediabetes group; ${ }^{b}<0.05$ versus normal group.

Abbreviations: $\gamma$-GT, $\gamma$-glutamyl transferase; ALP, alkaline phosphatase; ALT, alanine aminotransferase; apoB, apolipoprotein B; BMI, body mass index; CE, cholinesterase; DBP, diastolic blood pressure; FL, fatty liver; HDL-C, high-density lipoprotein cholesterol; HR, resting heart rate; LDL-C, low-density lipoprotein cholesterol; SBP, systolic blood pressure; SD, standard deviation; TC, total cholesterol; TG, triglyceride.

Table 3 Pearson univariate correlation analysis of metabolic syndrome components with insulin resistance and sensitivity index in type 2 diabetes mellitus

\begin{tabular}{|c|c|c|c|c|c|c|c|c|c|c|}
\hline \multirow[t]{2}{*}{ Value } & \multicolumn{2}{|c|}{ HOMA } & \multicolumn{2}{|c|}{ LogHOMA } & \multicolumn{2}{|c|}{ HOMA-1 $^{-1}$} & \multicolumn{2}{|c|}{ QUICKI } & \multicolumn{2}{|l|}{$\mathbf{I S I}_{0,120}$} \\
\hline & $r$ & $P$-value & $r$ & $P$-value & $r$ & $P$-value & $r$ & $P$-value & $r$ & $P$-value \\
\hline BMI & 0.407 & $0.000 *$ & 0.434 & $0.000^{*}$ & -0.328 & $0.000 *$ & -0.277 & $0.001 *$ & -0.262 & $0.002^{*}$ \\
\hline $\mathrm{HR}$ & 0.003 & 0.965 & 0.043 & 0.550 & -0.030 & 0.587 & -0.013 & 0.852 & 0.125 & 0.133 \\
\hline HT & 0.015 & $0.84 I$ & 0.050 & 0.493 & -0.083 & 0.251 & 0.029 & 0.687 & -0.077 & 0.357 \\
\hline SBP & 0.036 & 0.618 & 0.008 & 0.911 & 0.035 & 0.633 & 0.012 & 0.868 & 0.025 & 0.765 \\
\hline DBP & -0.005 & 0.945 & -0.022 & 0.765 & $0.04 I$ & 0.569 & 0.037 & 0.606 & 0.090 & 0.284 \\
\hline TC & 0.004 & 0.963 & 0.010 & 0.894 & 0.005 & 0.954 & 0.001 & 0.991 & -0.083 & 0.355 \\
\hline LDL-C & 0.006 & 0.935 & 0.022 & 0.779 & 0.010 & 0.894 & 0.009 & 0.911 & -0.110 & 0.222 \\
\hline HDL-C & -0.024 & 0.762 & -0.062 & 0.423 & 0.040 & 0.608 & -0.004 & 0.960 & -0.090 & 0.318 \\
\hline TG & 0.049 & 0.530 & 0.059 & 0.451 & -0.059 & 0.446 & -0.024 & 0.760 & -0.015 & 0.872 \\
\hline ApoB & -0.036 & 0.645 & -0.029 & 0.709 & 0.048 & $0.54 I$ & 0.026 & 0.737 & -0.040 & 0.656 \\
\hline FL & 0.181 & 0.089 & 0.225 & $0.034^{*}$ & -0.211 & $0.047^{*}$ & 0.011 & 0.916 & -0.186 & 0.110 \\
\hline ALT & $0.17 \mid$ & $0.02 I^{*}$ & 0.116 & 0.118 & -0.016 & 0.830 & -0.060 & 0.424 & -0.109 & 0.212 \\
\hline ALP & -0.064 & 0.393 & $-0.08 \mathrm{I}$ & 0.277 & 0.109 & 0.141 & 0.107 & 0.150 & 0.016 & 0.851 \\
\hline$\gamma$-GT & -0.004 & 0.955 & -0.015 & 0.846 & 0.057 & 0.448 & 0.012 & 0.873 & 0.008 & 0.928 \\
\hline CE & 0.093 & 0.238 & 0.128 & 0.102 & -0.113 & 0.152 & -0.007 & 0.929 & -0.118 & 0.206 \\
\hline
\end{tabular}

Note: $* p<0.05$.

Abbreviations: $\gamma$-GT, $\gamma$-glutamyl transferase; ALP, alkaline phosphatase; ALT, alanine aminotransferase; apoB, apolipoprotein B; BMI, body mass index; CE, cholinesterase; DBP, diastolic blood pressure; FL, fatty liver; HDL-C, high-density lipoprotein cholesterol; HOMA, homeostasis model assessment, an insulin resistance index; HOMA-1, reciprocal of HOMA, an insulin sensitivity index; HR, resting heart rate; $\mathrm{HT}$, hypertension; $\mathrm{ISI}_{0,120}$, Gutt's insulin sensitivity index; LDL-C, low-density lipoprotein cholesterol; LogHOMA, logarithm of HOMA, an insulin resistance index; QUICKI, quantitative insulin sensitivity check index; SBP, systolic blood pressure; TC, total cholesterol; TG, triglyceride.

Table 4 Logistic multivariate regression analysis of metabolic syndrome components with insulin resistance and sensitivity index in type 2 diabetes mellitus

\begin{tabular}{llllllll}
\hline Value & & $\beta$ & SE & Wald & P-value & Exp ( $\beta$ ) & 95\% CI \\
\hline HOMA & BMI & -0.491 & 0.222 & 4.890 & $0.027^{*}$ & 1.634 & $1.057-2.524$ \\
HOMA & ALT & -0.003 & 0.026 & 0.013 & 0.909 & 0.997 & $0.948-1.049$ \\
LogHOMA & BMI & -0.364 & 0.119 & 9.432 & $0.002^{*}$ & 0.695 & $0.551-0.877$ \\
LogHOMA & FL & 0.415 & 1.103 & 0.142 & 0.707 & 1.514 & $0.174-13.161$ \\
HOMA-1 $^{\text {HOMA-1 }}$ & BMI & -0.357 & 0.126 & 7.990 & $0.005^{*}$ & 0.700 & $0.547-0.896$ \\
QUICKI $_{\text {ISI }}$ & FL & -0.254 & 1.432 & 0.031 & 0.859 & 0.776 & $0.047-12.844$ \\
\hline
\end{tabular}

Note: $* \mathrm{P}<0.05$.

Abbreviations: $\mathrm{ALT}$, alanine aminotransferase; $\mathrm{BMI}$, body mass index; $\mathrm{Cl}$, confidence interval; $\mathrm{FL}$, fatty liver; HOMA, homeostasis model assessment, an insulin resistance index; $\mathrm{HOMA}^{-1}$, reciprocal of HOMA, an insulin sensitivity index; LogHOMA, logarithm of HOMA, an insulin resistance index; ISI ${ }_{0,120}$, Gutt's insulin sensitivity index; QUICKI, quantitative insulin sensitivity check index; SE, standard error. 


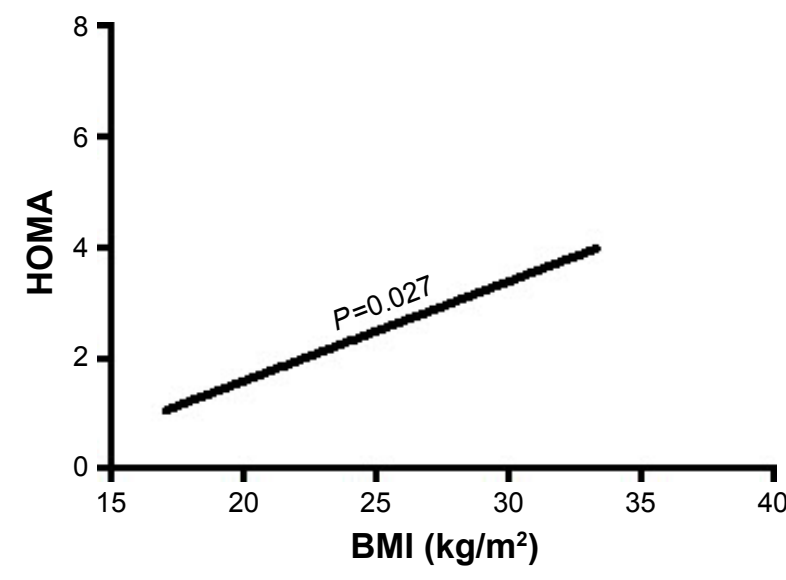

Figure I The association of BMI with HOMA.

Abbreviations: BMI, body mass index; HOMA, homeostasis model assessment, an insulin resistance index.

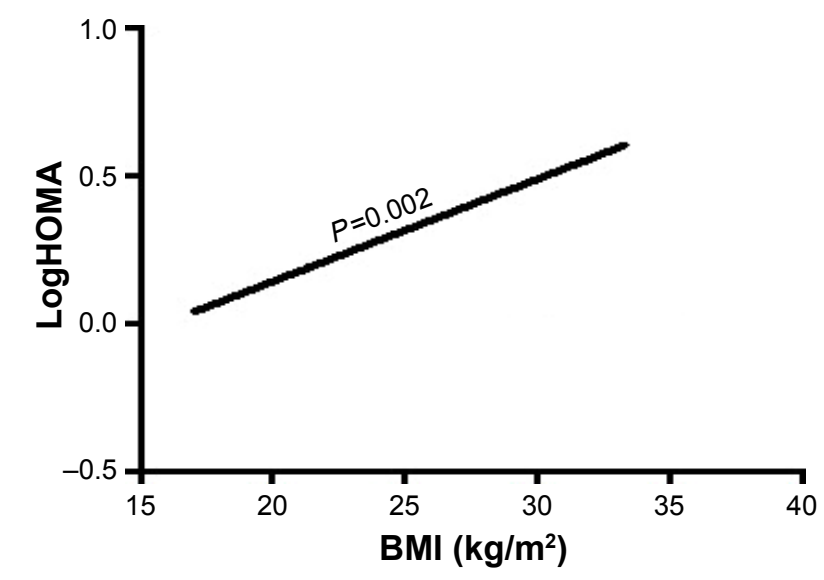

Figure 2 The association of BMI with LogHOMA.

Abbreviations: BMI, body mass index; HOMA, homeostasis model assessment, an insulin resistance index; LogHOMA, logarithm of HOMA, an insulin resistance index.

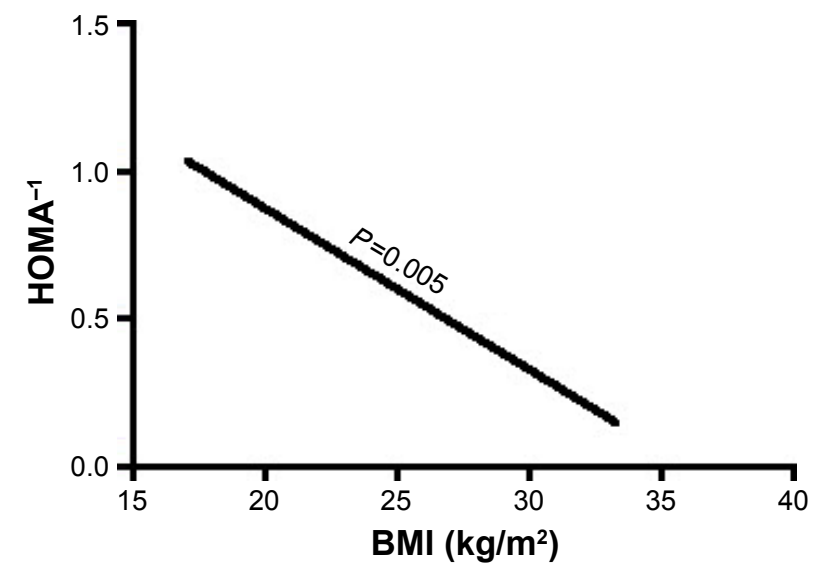

Figure 3 The association of BMI with $\mathrm{HOMA}^{-1}$.

Abbreviations: $\mathrm{BMI}$, body mass index; HOMA, homeostasis model assessment, an insulin resistance index; $\mathrm{HOMA}^{-1}$, reciprocal of HOMA, an insulin sensitivity index.

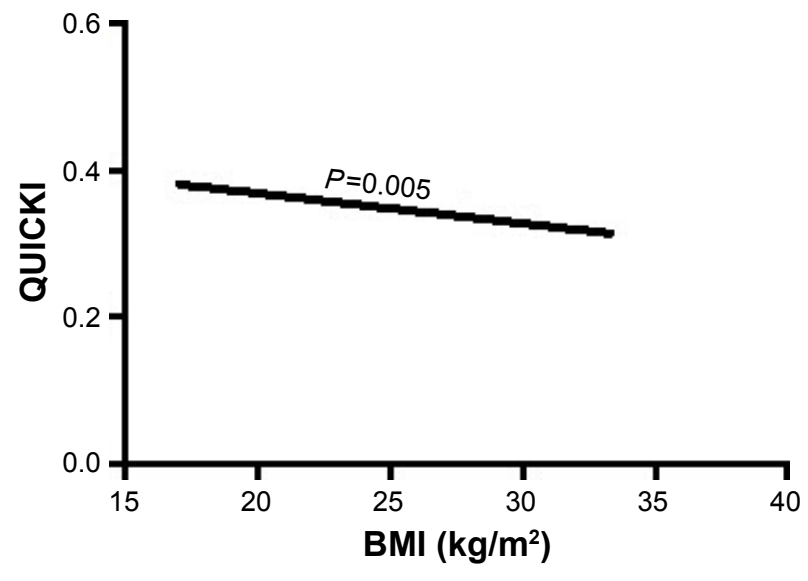

Figure 4 The association of BMI with insulin sensitivity index QUICKI.

Abbreviations: BMI, body mass index; QUICKI, quantitative insulin sensitivity check index.

Increased liver enzymes have been found to predict the risk of incident type $2 \mathrm{DM}$ in the elderly. ${ }^{27}$ Our present study identified that the prevalence of fatty liver and liver enzymes including ALT, ALP, $\gamma$-GT and cholinesterase were increased significantly with high insulin resistance and low insulin sensitivity in elderly patients with recently diagnosed type $2 \mathrm{DM}$. But this study revealed that fatty liver and liver enzymes were not independently associated with type $2 \mathrm{DM}$ in the elderly.

The major limitation of the present study is that the data were obtained retrospectively in a cross-sectional study by reviewing the patients' medical records. The confounding factors arising from the study setting, population selection, use of medications, exercise and dietary habits could not be completely excluded. The number of type $2 \mathrm{DM}$ patients among the elderly was quite limited. Although a previous study reported the association of higher BMI with

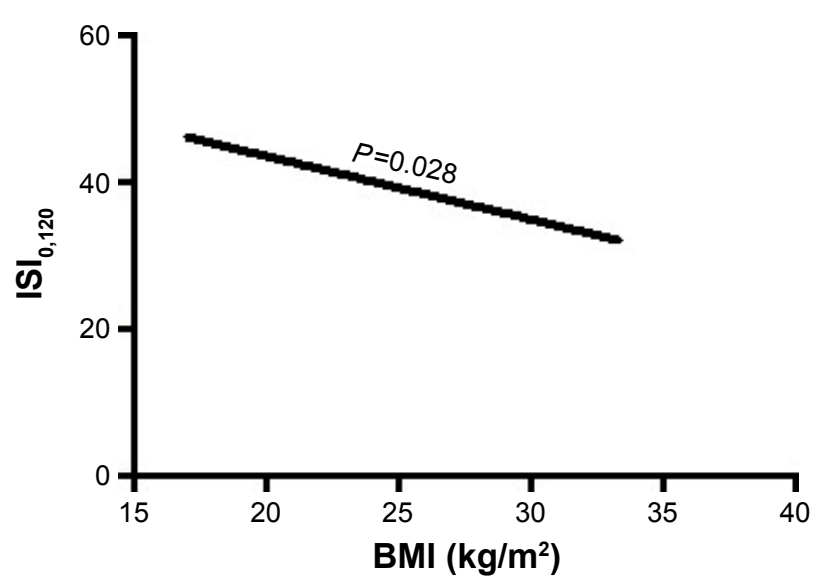

Figure 5 The association of BMI with insulin sensitivity index $\mathrm{ISI}_{0,120^{\circ}}$. Abbreviations: BMI, body mass index; $\mathrm{ISI}_{0,120}$, Gutt's insulin sensitivity index. 
prediabetes, ${ }^{28}$ our study did not support this conclusion. Therefore, large prospective study settings would be needed to confirm these findings.

\section{Conclusion}

Higher BMI was associated with increased insulin resistance and decreased insulin sensitivity in the elderly with type 2 DM.

\section{Acknowledgments}

This study was supported by the grants from National Key Clinical Specialties Construction Program of China (No [2013]544), the medical science and technology research fund of Health Bureau of Chongqing City, China (No 04-2154 and No 2009-2-290) and the natural science research fund of Chongqing Science and Technology Commission in Chongqing City, China (CSTC, No 2007BB5276).

\section{Disclosure}

The authors report no conflicts of interest in this work.

\section{References}

1. Zhao Y, Crimmins EM, Hu P, et al. Prevalence, diagnosis, and management of diabetes mellitus among older Chinese: results from the China Health and Retirement Longitudinal Study. Int J Public Health. 2016; 61(3):347-356.

2. Shaw JE, Sicree RA, Zimmet PZ. Global estimates of the prevalence of diabetes for 2010 and 2030. Diabetes Res Clin Pract. 2010; 87(1):4-14.

3. Imamura F, Mukamal KJ, Meigs JB, et al. Risk factors for type 2 diabetes mellitus preceded by beta-cell dysfunction, insulin resistance, or both in older adults: the Cardiovascular Health Study. Am J Epidemiol. 2013;177(12):1418-1429.

4. Novo S, Balbarini A, Belch JJ, et al. The metabolic syndrome: definition, diagnosis and management. Int Angiol. 2008;27(3):220-231.

5. Chen LK, Peng LN, Lin MH, et al. Predicting new onset diabetes mellitus in older Taiwanese metabolic syndrome or impaired fasting glucose? J Atheroscler Thromb. 2009;16(5):627-632.

6. Maggi S, Noale M, Gallina P, et al. Metabolic syndrome, diabetes, and cardiovascular disease in an elderly Caucasian cohort the Italian Longitudinal Study on Aging. J Gerontol A Biol Sci Med Sci. 2006;61(5): 505-510.

7. Noale M, Maggi S, Zanoni S, et al. The metabolic syndrome, incidence of diabetes and mortality among the elderly: the Italian Longitudinal Study of Ageing. Diabetes Metab. 2012;38(2):135-141.

8. Tanaka S, Tanaka S, Iimuro S, et al. Body mass index and mortality among Japanese patients with type 2 diabetes: pooled analysis of the Japan diabetes complications study and the Japanese elderly diabetes intervention trial. J Clin Endocrinol Metab. 2014;99(12):E2692-E2696.

9. Tobias DK, Pan A, Jackson CL, et al. Body-mass index and mortality among adults with incident type 2 diabetes. $N$ Engl J Med. 2014; 370(3):233-244.

10. Olafsdottir E, Aspelund T, Sigurdsson G, et al. Unfavourable risk factors for type 2 diabetes mellitus are already apparent more than a decade before onset in a population-based study of older persons: from the Age, Gene/Environment Susceptibility-Reykjavik Study (AGES-Reykjavik). Eur J Epidemiol. 2009;24(6):307-314.
11. Weiss A, Boaz M, Beloosesky Y, et al. Body mass index and risk of all-cause and cardiovascular mortality in hospitalized elderly patients with diabetes mellitus. Diabet Med. 2009;26(3):253-259.

12. Puavilai G, Chanprasertyotin S, Sriphrapradaeng A. Diagnostic criteria for diabetes mellitus and other categories of glucose intolerance: 1997 criteria by the Expert Committee on the Diagnosis and Classification of Diabetes Mellitus (ADA), 1998 WHO consultation criteria, and 1985 WHO criteria. World Health Organization. Diabetes Res Clin Pract. 1999;44(1):21-26.

13. Matthews DR, Hosker JP, Rudenski AS, et al. Homeostasis model assessment: insulin resistance and beta-cell function from fasting plasma glucose and insulin concentrations in man. Diabetologia. 1985; 28(7):412-419.

14. Chen H, Sullivan G, Quon MJ. Assessing the predictive accuracy of QUICKI as a surrogate index for insulin sensitivity using a calibration model. Diabetes. 2005;54(7):1914-1925.

15. Katz A, Nambi SS, Mather K, et al. Quantitative insulin sensitivity check index: a simple, accurate method for assessing insulin sensitivity in humans. J Clin Endocrinol Metab. 2000;85(7):2402-2410.

16. Gutt M, Davis CL, Spitzer SB, et al. Validation of the insulin sensitivity index (ISI $(0,120))$ : comparison with other measures. Diabetes Res Clin Pract. 2000;47(3):177-184.

17. Levey AS, Stevens LA, Schmid CH, et al. A new equation to estimate glomerular filtration rate. Ann Intern Med. 2009;150(9):604-612.

18. Lin SC, Heba E, Wolfson T, et al. Noninvasive diagnosis of nonalcoholic fatty liver disease and quantification of liver fat using a new quantitative ultrasound technique. Clin Gastroenterol Hepatol. 2015;13(7): 1337-1345, e1336.

19. Noale M, Maggi S, Marzari C, et al. Components of the metabolic syndrome and incidence of diabetes in elderly Italians: the Italian Longitudinal Study on Aging. Atherosclerosis. 2006;187(2):385-392.

20. Nagaya T, Yoshida H, Takahashi H, et al. Increases in body mass index, even within non-obese levels, raise the risk for type 2 diabetes mellitus: a follow-up study in a Japanese population. Diabet Med. 2005;22(8): 1107-1111.

21. Carnethon MR, Yan L, Greenland P, et al. Resting heart rate in middle age and diabetes development in older age. Diabetes Care. 2008;31(2): 335-339.

22. Emdin CA, Anderson SG, Woodward M, et al. Usual blood pressure and risk of new-onset diabetes: evidence from 4.1 million adults and a meta-analysis of prospective studies. J Am Coll Cardiol. 2015;66(14): 1552-1562.

23. Ley SH, Harris SB, Connelly PW, et al. Association of apolipoprotein B with incident type 2 diabetes in an aboriginal Canadian population. Clin Chem. 2010;56(4):666-670.

24. Beshara A, Cohen E, Goldberg E, et al. Triglyceride levels and risk of type 2 diabetes mellitus: a longitudinal large study. J Investig Med. 2016; 64(2):383-387.

25. Vega GL, Barlow CE, Grundy SM, et al. Triglyceride-to-high-densitylipoprotein-cholesterol ratio is an index of heart disease mortality and of incidence of type 2 diabetes mellitus in men. J Investig Med. 2014;62(2): 345-349.

26. Wang W, Liu J, Sun JY, et al. [The association between the changes in triglyceride levels and the risk of incident type 2 diabetes mellitus a 15 years followed-up results from the Chinese Multi-provincial Cohort Study]. Zhonghua Nei Ke Za Zhi. 2012;51(7):516-519.

27. Wannamethee SG, Shaper AG, Lennon L, et al. Hepatic enzymes, the metabolic syndrome, and the risk of type 2 diabetes in older men. Diabetes Care. 2005;28(12):2913-2918.

28. Serlachius A, Elovainio M, Juonala M, et al. The association between social support, body mass index and increased risk of prediabetes: the Cardiovascular Risk in Young Finns Study. Int J Behav Med. 2016. Epub 2016 Oct 3. 


\section{Publish your work in this journal}

Clinical Interventions in Aging is an international, peer-reviewed journal focusing on evidence-based reports on the value or lack thereof of treatments intended to prevent or delay the onset of maladaptive correlates of aging in human beings. This journal is indexed on PubMed Central, MedLine,

CAS, Scopus and the Elsevier Bibliographic databases. The manuscript management system is completely online and includes a very quick and fair peer-review system, which is all easy to use. Visit http://www.dovepress. com/testimonials.php to read real quotes from published authors. 